\title{
Physical Activity and Health-Related Quality of Life in Rural Adults with Chronic Disease
}

\author{
Peter D. Hart ${ }^{1,2,3, *}$ \\ ${ }^{1}$ Health Promotion Program, Montana State University - Northern, Havre, MT 59501 \\ ${ }^{2}$ Kinesmetrics Lab, Montana State University - Northern, Havre, MT 59501 \\ ${ }^{3}$ Health Demographics, Havre, MT 59501 \\ *Corresponding author: peter.hart@msun.edu
}

\begin{abstract}
Purpose: The purpose of this study was to examine the relationship between Physical activity (PA) and health-related quality of life (HRQOL) in a large-scale population of rural adults with chronic disease. Methods: Data for this study came from the CDC's 2015 Behavioral Risk Factor Surveillance System (BRFSS). A total of $\mathrm{N}=65,492$ rural adults $20+$ years of age were included in the analysis. The main outcome variable was HRQOL as assessed by the CDC Healthy Days Index. Adults were categorized as "meeting" PA guidelines if they reported 150+ minutes of moderate-intensity PA per week and "not meeting" PA guidelines if they reported less than 150 minutes. Multiple logistic regression was used to compute odds ratios (ORs) and 95\% confidence intervals (CIs) while adjusting for age, sex, race, and income. Results: Rural adults with at least one chronic disease and meeting recommended levels of PA were significantly more likely to report good HRQOL (72.5\%; 70.7-74.3) than their less active counterparts (58.1\%; 56.4-59.7, $\mathrm{p}<.001$ ). Those meeting recommended levels of PA had greater odds of good HRQOL regardless of reporting diabetes (OR=1.58; 95\% CI: 1.31-1.91), COPD (1.49; 1.20-1.85), cancer $(1.79 ; 1.43-2.22)$, stroke $(1.73 ; 1.30-2.31)$, heart disease $(1.52 ; 1.18-1.96)$, or heart attack $(1.68 ; 1.32-2.15)$. Additionally, the same increased odds were seen across rural adults reporting 1 (1.59; 1.34-1.88), 2 (1.64; 1.28-2.11), and 3+ (1.52; 1.13-2.05) chronic diseases. Conclusions: Results from this study indicate that meeting recommended levels of PA is strongly associated with optimal levels of HRQOL among rural adults with chronic disease.
\end{abstract}

Keywords: physical activity, chronic disease, epidemiology, health-related quality of life

Cite This Article: Peter D. Hart, "Physical Activity and Health-Related Quality of Life in Rural Adults with Chronic Disease.” American Journal of Medical Sciences and Medicine, vol. 5, no. 3 (2017): 62-66. doi: 10.12691/ajmsm-5-3-5.

\section{Introduction}

Adults residing in rural regions of the Unites States (U.S.) are known to suffer from poorer health outcomes than their urban counterparts [1]. Prevalence of diabetes and coronary heart disease in rural populations is higher than other geographic regions [2]. Rural residents are also significantly more likely to die after traumatic injury, as compared to their non-rural counterparts [3]. These health disparities are also seen with health risk behaviors. The prevalence of good health behaviors (i.e., sufficient sleep, non-smoking, non-alcohol drinking, normal body weight maintenance, and meeting physical activity guidelines) is also lower in rural regions [4]. Access to proper and appropriate healthcare may be one factor explaining such rural health disparities, with rural residents suffering less quality access to healthcare providers $[5,6]$.

Physical activity (PA), a health behavior associated with many positive health outcomes, is also associated with health-related quality of life (HRQOL) in adults [7]. Furthermore, adults with chronic disease [8] and adults residing in rural regions [9] suffer disparities in HRQOL. Given this, however, little is known regarding the association between PA and HRQOL in rural adults suffering from chronic illness. Therefore, purpose of this study was to examine the relationship between PA and HRQOL in a large-scale population of rural adults with chronic disease.

\section{Methods}

\subsection{Participants and Design}

Data for this study came from the CDC's 2015 Behavioral Risk Factor Surveillance System (BRFSS), a large national cross-sectional survey [10]. The BRFSS is a state-based annual survey of U.S. non-institutionalized adults 18+ years of age. The BRFSS uses a complex probability sampling scheme and provides estimates that are representative of all U.S. adults. The BRFSS is designed to monitor risky health behaviors and chronic conditions associated with the nation's leading causes of death and disease. The 2015 BRFSS included survey questions regarding fifteen core sections: 1) demographics, 2) health status, 3) health-related quality of life, 4) health care access, 5) hypertension, 6) cholesterol, 7) chronic health, 8) tobacco use, 9) alcohol consumption, 10) fruit and vegetable consumption, 11) physical activity, 12) arthritis, 
13) seatbelt use, 14) immunization, and 15) HIV/AIDS [11]. This study used participant responses primarily from the demographics, HRQOL, chronic health, and physical activity cores. A total of $\mathrm{N}=65,492$ adults $20+$ years of age, who answered all relevant survey questions, and resided in a rural U.S. county were included [12].

\subsection{Measures}

HRQOL was the outcome variable for all analyses. The main independent variable was PA status (meeting or not meeting guidelines). Stratification variables consisted of chronic disease indicators of diabetes, COPD, cancer, heart disease, stroke, or heart attack. HRQOL was assessed by the CDC Healthy Days Index. Adults reporting 13 or fewer unhealthy days were considered to have "good" HRQOL and those reporting 14 or more unhealthy days were considered to have "poor" HRQOL [13]. PA was assessed based on responses to a series of questions regarding $\mathrm{PA}$ in the past 30 days. Participants reporting $150+$ minutes of moderate-intensity PA per week were considered to have met the PA guidelines [14].

Participants were considered having a chronic disease if they reported ever being told by a health professional that they had diabetes, COPD, cancer, heart disease, a stroke

or a heart attack. The variables age, sex, race, and income were used to describe the data as well as for covariates in adjusted models. Body mass index (BMI) was used as an additional covariate and computed from self-reported height and weight. Those respondents with BMIs less than $25\left(\mathrm{~kg} / \mathrm{m}^{2}\right)$ were considered normal weight, those with BMIs greater than or equal to 25 but less than $30\left(\mathrm{~kg} / \mathrm{m}^{2}\right)$ were considered overweight and those with BMIs greater than or equal to $30\left(\mathrm{~kg} / \mathrm{m}^{2}\right)$ were considered obese.

\subsection{Statistical Analysis}

Prevalence estimates (\%s) were computed for both HRQOL and PA status across relevant sociodemographic groups. Chi-square tests of independence were used to test for significant differences in prevalence estimates. Multiple logistic regression was used to compute odds ratios (ORs) and 95\% confidence intervals (CIs) while adjusting for age, sex, race, and income [15,16]. SPSS Complex Sampling version 24 and SAS version 9.4 were used to account for the sampling design [17,18]. All significance levels were set to $\mathrm{p}=.05$.

\section{Results}

Table 1. Prevalence of meeting and not meeting recommended levels of PA and good and poor HRQOL among rural adults with at least one chronic disease by demographic category, 2015

\begin{tabular}{|c|c|c|c|c|c|c|c|c|c|c|}
\hline & \multicolumn{4}{|c|}{ Met PA Guidelines } & \multirow[b]{3}{*}{$\mathrm{p}$} & \multicolumn{4}{|c|}{ Reports Good HRQOL } & \multirow[b]{3}{*}{$p$} \\
\hline & \multicolumn{2}{|r|}{ Yes } & \multicolumn{2}{|r|}{ No } & & \multicolumn{2}{|c|}{ Yes } & \multicolumn{2}{|c|}{ No } & \\
\hline & $\%$ & $95 \%$ CI & $\%$ & $95 \%$ CI & & $\%$ & $95 \% \mathrm{CI}$ & $\%$ & $95 \%$ CI & \\
\hline Overall & 41.3 & $40.2-42.5$ & 58.7 & $57.5-59.8$ & $<.001$ & 64.3 & $63.1-65.4$ & 35.7 & $34.6-36.9$ & $<.001$ \\
\hline Gender & & & & & $<.001$ & & & & & $<.001$ \\
\hline Male & 46.1 & $44.3-48.0$ & 53.9 & $52.0-55.7$ & & 68.0 & $66.2-69.7$ & 32.0 & 30.3-33.8 & \\
\hline Female & 37.7 & $36.2-39.2$ & 62.3 & $60.8-63.8$ & & 61.4 & 59.9-62.9 & 38.6 & $37.1-40.1$ & \\
\hline Age Group (yr.) & & & & & .001 & & & & & $<.001$ \\
\hline $20-24$ & 53.3 & $32.8-72.7$ & 46.7 & $27.3-67.2$ & & 70.5 & $47.4-86.4$ & 29.5 & $13.6-52.6$ & \\
\hline $25-34$ & 44.2 & $33.0-56.1$ & 55.8 & $43.9-67.0$ & & 53.7 & $42.2-64.8$ & 46.3 & $35.2-57.8$ & \\
\hline $35-44$ & 42.8 & 36.2-49.6 & 57.2 & $50.4-63.8$ & & 54.9 & 48.6-61.1 & 45.1 & 38.9-51.4 & \\
\hline $45-54$ & 37.1 & 33.6-40.8 & 62.9 & $59.2-66.4$ & & 51.5 & $47.8-55.2$ & 48.5 & $44.8-52.2$ & \\
\hline $55-64$ & 36.9 & 34.6-39.3 & 63.1 & $60.7-65.4$ & & 59.5 & $57.1-61.8$ & 40.5 & $38.2-42.9$ & \\
\hline $65+$ & 43.9 & $42.4-45.4$ & 56.1 & $54.6-57.6$ & & 70.4 & $69.0-71.8$ & 29.6 & $28.2-31.0$ & \\
\hline Race/Ethnicity & & & & & $<.001$ & & & & & .135 \\
\hline White & 42.5 & $41.2-43.8$ & 57.5 & $56.2-58.8$ & & 64.9 & 63.7-66.1 & 35.1 & 33.9-36.3 & \\
\hline Black & 28.4 & $24.5-32.6$ & 71.6 & $67.4-75.5$ & & 62.8 & $58.3-67.2$ & 37.2 & $32.8-41.7$ & \\
\hline Hispanic & 42.1 & $32.8-52.0$ & 57.9 & $48.0-67.2$ & & 59.4 & 49.7-68.4 & 40.6 & $31.6-50.3$ & \\
\hline Multiracial & 40.2 & $32.5-48.3$ & 59.8 & $51.7-67.5$ & & 54.1 & $45.3-62.7$ & 45.9 & $37.3-54.7$ & \\
\hline Other & 40.3 & $33.8-47.1$ & 59.7 & 52.9-66.2 & & 60.2 & $53.5-66.6$ & 39.8 & $33.4-46.5$ & \\
\hline Education & & & & & $<.001$ & & & & & $<.001$ \\
\hline$<$ High School & 29.8 & $27.0-32.9$ & 70.2 & $67.1-73.0$ & & 56.8 & $53.7-60.0$ & 43.2 & $40.0-46.3$ & \\
\hline High School Graduate & 39.5 & $37.7-41.4$ & 60.5 & $58.6-62.3$ & & 65.0 & $63.3-66.7$ & 35.0 & $33.3-36.7$ & \\
\hline Some College & 46.7 & $44.5-49.0$ & 53.3 & $51.0-55.5$ & & 64.2 & $62.0-66.3$ & 35.8 & $33.7-38.0$ & \\
\hline College Graduate & 55.5 & $53.1-57.8$ & 44.5 & $42.2-46.9$ & & 75.6 & $73.5-77.7$ & 24.4 & $22.3-26.5$ & \\
\hline Marital Status & & & & & $<.001$ & & & & & $<.001$ \\
\hline Married & 44.2 & $42.5-45.8$ & 55.8 & $54.2-57.5$ & & 67.2 & $65.6-68.7$ & 32.8 & $31.3-34.4$ & \\
\hline Not Married & 37.3 & 35.6-39.0 & 62.7 & $61.0-64.4$ & & 60.2 & $58.4-61.8$ & 39.8 & $38.2-41.6$ & \\
\hline Income (US \$) & & & & & $<.001$ & & & & & $<.001$ \\
\hline$<15,000$ & 33.3 & 29.9-36.9 & 66.7 & 63.1-70.1 & & 43.6 & $53.0-59.8$ & 56.4 & $53.0-59.8$ & \\
\hline $15-24,999$ & 36.1 & 33.6-38.6 & 63.9 & 61.4-66.4 & & 59.2 & $56.6-61.8$ & 40.8 & $38.2-43.4$ & \\
\hline 25-34,999 & 41.5 & $38.0-45.1$ & 58.5 & $54.9-62.0$ & & 63.9 & $60.3-67.4$ & 36.1 & 32.6-39.7 & \\
\hline $35-49,999$ & 46.9 & $43.6-50.3$ & 53.1 & $49.7-56.4$ & & 72.0 & 68.7-75.1 & 28.0 & 24.9-31.3 & \\
\hline $50,000+$ & 53.3 & $50.9-55.7$ & 46.7 & 44.3-49.1 & & 77.0 & $75.0-79.0$ & 23.0 & $21.0-25.0$ & \\
\hline
\end{tabular}

Note. p-values are for the Rao-Scott chi-square statistic. 150+ minutes of moderate-intensity PA (or vigorous equivalent) was guideline used for meeting PA. Adults reporting 13 or fewer unhealthy days were considered to have "good" HRQOL and those reporting 14 or more unhealthy days were considered to have "poor" HRQOL. 
Table 2. Odds of Good HRQOL for Rural Adults Meeting Recommended Levels of PA

\begin{tabular}{|c|c|c|c|c|}
\hline \multirow[b]{3}{*}{ Chronic Disease State } & \multirow{2}{*}{\multicolumn{2}{|c|}{ Unadjusted }} & \multirow{2}{*}{\multicolumn{2}{|c|}{ Adjusted }} \\
\hline & & & & \\
\hline & OR & $95 \%$ CI & OR & $95 \%$ CI \\
\hline Diabetes & 1.81 & $1.53-2.14$ & 1.58 & $1.31-1.91$ \\
\hline COPD & 1.70 & $1.40-2.07$ & 1.49 & $1.20-1.85$ \\
\hline Cancer & 2.05 & $1.69-2.49$ & 1.79 & $1.43-2.22$ \\
\hline Stroke & 1.71 & $1.29-2.26$ & 1.73 & $1.30-2.31$ \\
\hline Heart Disease & 1.86 & $1.49-2.33$ & 1.52 & $1.18-1.96$ \\
\hline Heart Attack & 1.97 & $1.58-2.46$ & 1.68 & $1.32-2.15$ \\
\hline Has 1 Chronic Disease & 1.82 & $1.57-2.112$ & 1.59 & $1.34-1.88$ \\
\hline Has 2 Chronic Diseases & 1.93 & $1.54-2.42$ & 1.64 & $1.28-2.11$ \\
\hline Has 3+ Chronic Diseases & 1.79 & $1.35-2.36$ & 1.52 & $1.13-2.05$ \\
\hline
\end{tabular}

Note. Adjusted OR are adjusted for age, sex, race, and income.

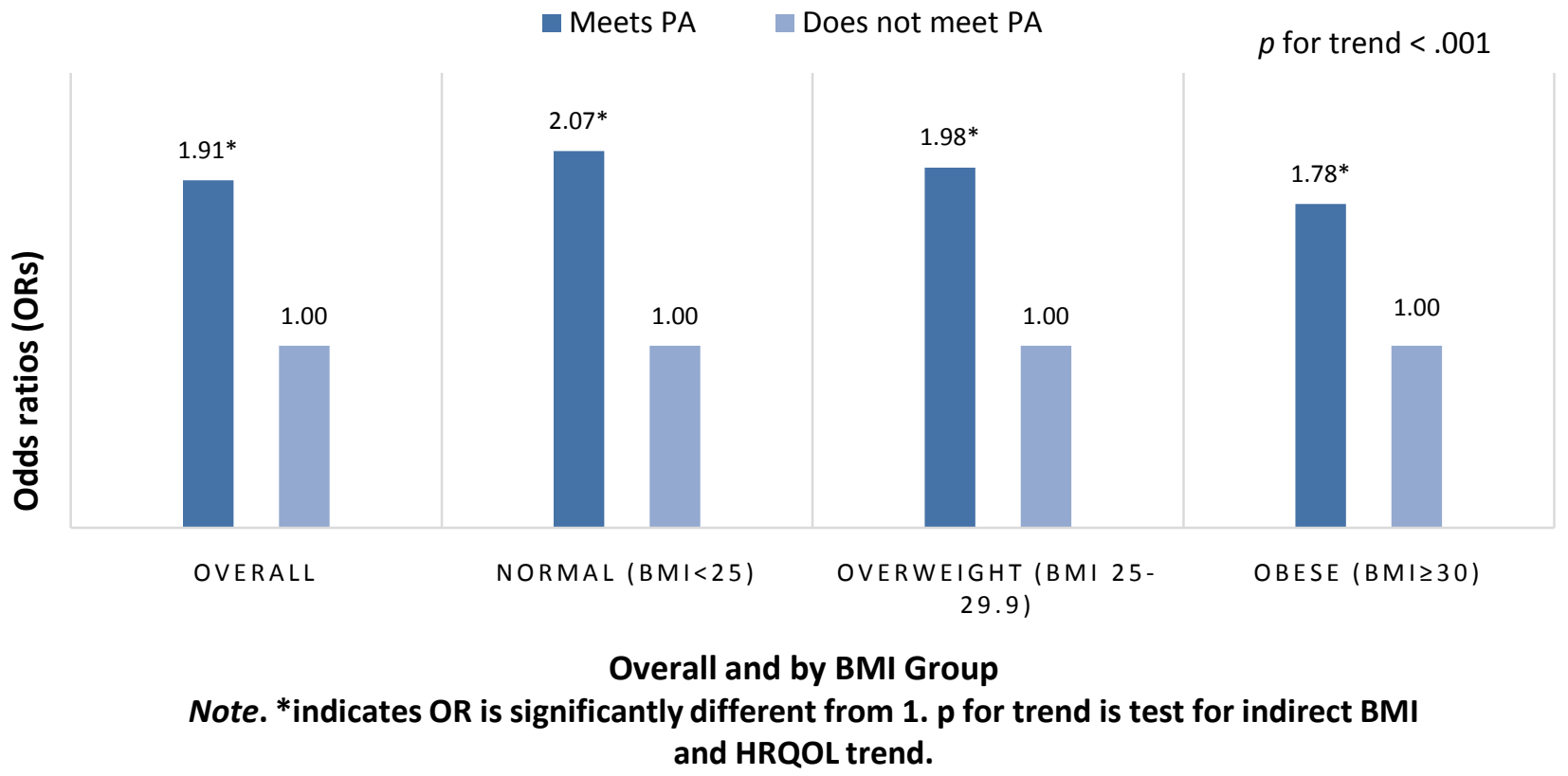

Figure 1. Odds ratios (ORs) of good HRQOL for those meeting recommended levels of PA across BMI categories

Table 1 displays prevalence estimates for good/poor HRQOL and PA status. Majority of rural adults did not meet PA guidelines (58.7\%; 57.5-59.8), with more males (46.1\%; 44.3-48.0) meeting guidelines than females (37.7\%; 36.2-39.2). However, majority of rural adults did report at least good HRQOL (64.3\%; 63.1-65.4), with more females (38.6\%; 37.1-40.1) reporting poor HRQOL than males (32.0\%; 30.3-33.8). Rural adults with at least one chronic disease and meeting recommended levels of PA were significantly more likely to report good HRQOL (72.5\%; 70.7-74.3) than their less active counterparts (58.1\%; 56.4-59.7, $\mathrm{p}<.001$ ).

Table 2 displays odds of good HRQOL (as compared to poor HRQOL) for rural adults meeting PA guidelines (as compared to not meeting guidelines) across chronic disease status. Those meeting recommended levels of PA had greater odds of good HRQOL regardless of reporting diabetes (OR=1.58; 95\% CI: 1.31-1.91), COPD (1.49; $1.20-1.85)$, cancer $(1.79 ; 1.43-2.22)$, stroke $(1.73 ; 1.30-$ 2.31), heart disease $(1.52 ; 1.18-1.96)$, or heart attack (1.68;
1.32-2.15), in the adjusted models. Additionally, the same increased odds were seen across rural adults reporting 1 (1.59; 1.34-1.88), 2 (1.64; 1.28-2.11), and 3+ (1.52; 1.132.05) chronic diseases.

Figure 1 displays the ORs and associated significance tests for PA as a predictor of HRQOL, overall and by BMI category. Overall, those meeting PA guidelines were more likely $(1.91 ; \mathrm{p}<.05)$ to report good HRQOL, as compared to their less active counterparts. Rural adults of normal body weight had the greatest odds $(2.07 ; \mathrm{p}<.05)$ of good HRQOL given they met PA guidelines and obese adults had the lowest $(1.78 ; \mathrm{p}<.05)$. Additionally, the odds associated with good HRQOL were indirectly related to BMI category (p for trend <.001).

\section{Discussion}

The purpose of this study was to examine the relationship between PA and HRQOL in a large-scale 
population of rural adults with chronic disease. The results clearly showed that meeting recommended levels of PA was indeed related to optimal levels of HRQOL, despite chronic disease status. These findings remained robust across the different chronic disease conditions (i.e., diabetes, cancer, stroke, etc.). Moreover, adults with multiple chronic illnesses appeared to receive similar benefit from recommended levels of PA. These results are consistent with randomized controlled trials that have shown increases in PA with simultaneous increases in HRQOL [19,20,21,22]. These findings are further supported by studies that have shown increased HRQOL in physically active participants with chronic diseases [23-29]. Finally, these results are consistent with studies that have shown significant relationships between PA and HRQOL among rural adults suffering from chronic illness [30,31].

The strengths concerning this study are worth mentioning. This study analyzed data that are representative of all U.S. rural adults $20+$ years of age. Few studies researching similar PA and HRQOL relationships in this population allow for this type of generalization. Another strength was the ability to assess chronic disease states from survey data. The BRFSS is a rich survey in that it assesses several different health conditions. Therefore, this study had the ability to assign each participant a chronic disease status as well as assess the number of chronic diseases each participant reported.

This study has limitations worth mentioning before the results are completely interpreted. This study was limited to participants self-reporting whether a healthcare professional had ever told them they had a chronic disease condition. This type of assessment may introduce measurement error both in terms of participant recall accuracy as well as severity of the chronic health condition. The BRFSS questions regarding chronic conditions do not differentiate between type of healthcare provider and severity of the disease. Therefore, some participants may have been misclassified as having the condition. A clinical-based study, that provides medical examinations to subjects, may provide a more accurate chronic disease classification. Similarly, because this study used self-report data for chronic disease assessment, it's possible that some participants suffered from certain chronic conditions during their interview but were, however, unaware they had a condition. These limitations warrant caution when interpreting the findings from this study.

\section{Conclusions}

Results from this study indicate that meeting recommended levels of PA is strongly associated with optimal levels of HRQOL among rural adults with chronic disease. Health promotion programs should market PA interventions to rural adults with such diseases to improve HRQOL.

\section{Acknowledgements}

No financial assistance was used to assist with this project.

\section{References}

[1] Matthews KA. Racial/Ethnic Health Disparities Among Rural Adults - United States, 2012-2015. MMWR. Surveillance Summaries. 2017; 66.

[2] O'Connor A, Wellenius G. Rural-urban disparities in the prevalence of diabetes and coronary heart disease. Public health. 2012 Oct 31; 126(10): 813-20.

[3] Jarman MP, Castillo RC, Carlini AR, Kodadek LM, Haider AH. Rural risk: Geographic disparities in trauma mortality. Surgery. 2016 Dec 31; 160(6): 1551-9.

[4] Matthews KA. Health-related behaviors by Urban-Rural County Classification-United States, 2013. MMWR. Surveillance Summaries. 2017; 66.

[5] Carr BG, Bowman AJ, Wolff CS, Mullen MT, Holena DN, Branas CC, Wiebe DJ. Disparities in access to trauma care in the United States: a population-based analysis. Injury. 2017 Feb 28; 48(2): 332-8.

[6] Davis MM, Spurlock M, Dulacki K, Meath T, Li HF, McCarty D, Warne D, Wright B, McConnell KJ. Disparities in Alcohol, Drug Use, and Mental Health Condition Prevalence and Access to Care in Rural, Isolated, and Reservation Areas: Findings From the South Dakota Health Survey. The Journal of Rural Health. 2016 Jun 1; 32(3): 287-302.

[7] Hart, P. D. (2016). Meeting recommended levels of physical activity and health-related quality of life in rural adults. Journal of Lifestyle Medicine, 6(1): 1-6.

[8] Hart, P. D., Benavidez, G. A., \& Erickson, J. (2017). Meeting recommended levels of physical activity in relation to preventive health behavior and health status among adults. Journal of Preventive Medicine and Public Health. 2017; 50(1): 10-17..

[9] Miles, A., Proescholdbell, R. J., \& Puffer, E. (2011). Explaining rural/non-rural disparities in physical health-related quality of life: a study of United Methodist clergy in North Carolina. Quality of Life Research, 20(6), 807-815.

[10] Centers for Disease Control and Prevention (CDC). Behavioral Risk Factor Surveillance System Survey Data. Atlanta, Georgia: U.S. Department of Health and Human Services, Centers for Disease Control and Prevention; 2015.

[11] Centers for Disease Control and Prevention (CDC). Behavioral Risk Factor Surveillance System Questionnaire. Atlanta, Georgia: U.S. Department of Health and Human Services, Centers for Disease Control and Prevention; 2015.

[12] Swanoski MT, Lutfiyya MN, Amaro ML, Akers MF, Huot KL. (2012). Knowledge of heart attack and stroke symptomology: a cross-sectional comparison of rural and non-rural US adults. BMC Public Health. 2012; 12, article 283.

[13] Centers for Disease Control and Prevention (CDC). Measuring healthy days: Population assessment of health-related quality of life. Atlanta, Georgia: CDC; 2000.

[14] Centers for Disease Control and Prevention. A data users guide to the BRFSS physical activity questions: how to assess the 2008 physical activity guidelines for Americans.

[15] Allison PD. Survival analysis using SAS: a practical guide. SAS Institute; 2010.

[16] Stokes, M. E., Davis, C. S., \& Koch, G. G. (2012). Categorical data analysis using SAS. SAS institute.

[17] IBM Corporation. IBM SPSS Complex Samples: Correctly Compute Complex Samples Statistics. Somers, NY: IBM; 2012.

[18] Cody, R. P., \& Smith, J. K. (2006). Applied statistics and the SAS programming language. 5th Edition. Pearson.

[19] Jahangiry L, Montazeri A, Najafi M, Yaseri M, Farhangi MA. An interactive web-based intervention on nutritional status, physical activity and health-related quality of life in patient with metabolic syndrome: a randomized-controlled trial (The Red Ruby Study). Nutrition \& diabetes. 2017 Jan 1; 7(1): e240.

[20] Mansikkamäki K, Raitanen J, Nygård CH, Tomás E, Rutanen R, Luoto R. Long-term effect of physical activity on health-related quality of life among menopausal women: a 4-year follow-up study to a randomised controlled trial. BMJ open. 2015 Sep 1; 5(9): e008232.

[21] Olsson SJ, Börjesson M, Ekblom-Bak E, Hemmingsson E, Hellénius ML, Kallings LV. Effects of the Swedish physical activity on prescription model on health-related quality of life in overweight older adults: a randomised controlled trial. BMC public health. 2015 Jul 21; 15(1): 687. 
[22] Brovold T, Skelton DA, Bergland A. Older Adults Recently Discharged from the Hospital: Effect of Aerobic Interval Exercise on Health-Related Quality of Life, Physical Fitness, and Physical Activity. Journal of the American Geriatrics Society. 2013 Sep 1; 61(9): 1580-5.

[23] Thiel DM, Al Sayah F, Vallance J, Johnson ST, Johnson JA Physical Activity and Health-Related Quality of Life in Adults With Type 2 Diabetes: Results From a Prospective Cohort Study. Journal of Physical Activity and Health. 2017: 1-23.

[24] Thiel DM, Al Sayah F, Vallance JK, Johnson ST, Johnson JA Association between physical activity and health-related quality of life in adults with type 2 diabetes. Canadian journal of diabetes. 2017 Feb 28; 41(1): 58-63.

[25] Esteban C, Quintana JM, Aburto M, Moraza J, Egurrola M, PérezIzquierdo J, Aizpiri S, Aguirre U, Capelastegui A. Impact of changes in physical activity on health-related quality of life among patients with COPD. European Respiratory Journal. 2010 Aug 1; 36(2): 292-300.

[26] Rand D, Eng JJ, Tang PF, Hung C, Jeng JS. Daily physical activity and its contribution to the health-related quality of life of ambulatory individuals with chronic stroke. Health and quality of life outcomes. 2010 Aug 3; 8(1): 80.
[27] Shin WK, Song S, Jung SY, Lee E, Kim Z, Moon HG, Noh DY, Lee JE. The association between physical activity and healthrelated quality of life among breast cancer survivors. Health and quality of life outcomes. 2017 Jun 30; 15(1): 132.

[28] Awotidebe TO, Adeyeye VO, Ogunyemi SA, Bisiriyu LA, Adedoyin RA, Balogun MO, Adebayo RA, Amosun OD. Joint predictability of physical activity and body weight status on health-related quality of life of patients with hypertension. Journal of Exercise Rehabilitation. 2017 Oct; 13(5): 588.

[29] Austin S, Qu H, Shewchuk RM. Association between adherence to physical activity guidelines and health-related quality of life among individuals with physician-diagnosed arthritis. Quality of Life Research. 2012 Oct 1; 21(8): 1347-57.

[30] Hageman PA, Pullen CH, Yoerger M. Physical Function and Health-Related Quality of Life in Overweight and Obese Rural Women who Meet Physical Activity Recommendations. Journal of Aging and Physical Activity. 2017: 1-37.

[31] Vallance JK, Lavallee CM, Culos-Reed NS, Trudeau MG. Physical activity is associated with clinically important differences in health-related quality of life among rural and small-town breast cancer survivors. Supportive Care in Cancer. 2012 May 1; 20(5): 1079-87. 